\title{
Research on medical image processing based on ITK algorithm
}

\author{
Wang Jinguo ${ }^{1, a}$, Wang $\mathrm{Na}^{2, b^{*}}$ (corresponding author), Wang rui ${ }^{3, \mathrm{c}}$ \\ ${ }^{1}$ Department of Urology, the First Hospital of Jilin University, China \\ 2Department of Anesthesiology, the First Hospital of Jilin University, China \\ ${ }^{3}$ Department of Anesthesiology, the First Hospital of Jilin University, China \\ a'wangjinguolily@163.com, ${ }^{\mathrm{b}}$ lilyly12345@163.com, ${ }^{\mathrm{C} X i a o b e n 6666 @ 126 . c o m ~}$
}

Keywords: ITK algorithm. Medical image processing.

Abstract. With the development of advanced medical imaging equipment and image processing algorithms, medical imaging technology has been developed rapidly, and medical diagnosis and treatment technology has made considerable progress. Now, doctors have been able to use medical imaging to see the patient's lesion area, you can see a series of tomographic images from a multi-angle and multi-dimensional approach to observe the lesion area. By these imaging methods, the patient's pain and the accuracy of diagnosis were improved.

\section{Introduction}

Application of computer to assist doctors in the diagnosis and analysis of medical imaging technology, called medical image processing and analysis. They can help doctors better, more convenient and more accurate diagnosis[1]. Medical image processing and analysis includes many aspects: image processing, computer graphics, pattern recognition, virtual reality, medical knowledge, etc.[2]. At present, the most popular research fields include medical image segmentation, medical image registration, medical image information fusion, and three-dimensional visualization of four aspects[3][4].

Medical image segmentation is to separate the different regions of medical images with special meaning, so that each region can meet the consistency of a specific region[5]. It is a basic problem in image processing and image analysis. At present, a number of image segmentation algorithms have been proposed for various specific problems, and the results of image segmentation are also very good. However, because of the special nature of medical image segmentation problem, it has not been successful, and has a universal solution[6].

Medical images may come from different types of acquisition equipment or different collection time, in order to accurately analyze the image, image registration is very important. Medical image registration is to find a space transformation, so that the corresponding point of the two images to achieve the same space position and anatomical structure. The structure of the registration is required to make all of the anatomic points on the two images, or at least all of the diagnostic significance and surgical area of the point to achieve the matching[7].

Image registration is the premise of image fusion, it is recognized that the difficulty of image processing technology, but also the key technology to determine the development of medical image fusion technology. In recent years at home and abroad in terms of image registration, such as geometric moments of registration based on mutual information registration, pyramid multi-level registration, the correlation coefficient of the image, the spline interpolation of image registration. 


\section{Medical image segmentation algorithm based on ITK}

Although there are a lot of work on medical image segmentation, there is no single method can be used to solve a large number of different types of medical image segmentation problem. The most effective way is to combine a lot of different methods. Different components of the parameters for different types of image features and anatomical structure to set up, in order to carry out segmentation.

ITK provides a number of basic algorithms that can be used to develop and tailor the segmentation application into practice.

Region growing method. Region growing algorithm has been proved to be an effective method for image segmentation. The basic algorithm of region growing is to select a seed region, which is considered to be in the target region for the segmentation. The neighborhood of the pixel is judged by a method in the target region, if the region is added to the seed region, and the process is continued until no new point is added to the target area. The difference of regional growth algorithm is mainly the difference of the criterion. We take confidence connected as an example, and implement the region growing algorithm in ITK.

Its judging criterion is based on the statistical properties of the simple current region. Firstly, the mean and standard deviation of the gray value of all the pixels in the region are calculated. The user defines a product factor, which is multiplied by the standard deviation, and defines the range of a mean value. The criterion for judging the algorithm is:

$$
I(x) \in[m-f \sigma, m+f \sigma]
$$

In which, $\mathrm{m}$ is average. $\sigma$ is standard deviation. $f$ is product factor. If the gray value of the pixels in the neighborhood is within the region, it can be accepted by the region. When there is no new field pixel to meet the criterion of judgment, the algorithm considers that it is over. At this time, the mean and standard deviation in the new area, and then repeat the above steps from the new area. This iterative process is repeated until no new pixels are added or the maximum number of iterations has been reached.

level set segmentation. The set Level method is a numerical method for tracking the contour and surface evolution. It is not directly on the contour to operate, and the zero level set of the contour as a function of a high dimensional function is contained in it. We define the level-set function is $\varphi(x, t)$. The function is evolution controlled by different equations. At any given moment, the evolution of the contour can be obtained by extracting the zero level set of the level set function.

$$
\Gamma((x), t)=\{\varphi(x, t)=0\}
$$

We use Level-set of the ITK method to achieve medical image segmentation. Each of these filters is a general Level-set equation to calculate the solution of the partial differential equation.

$$
\frac{d}{d t} \varphi=-\alpha A(x) \cdot \nabla \varphi-\beta p(x)|\nabla \varphi|+\gamma Z(x)|\nabla \varphi|
$$

In which, $\mathrm{A}$ is a horizontal convection term. $\mathrm{P}$ is a communication item. $\mathrm{Z}$ is a local adjustment item. The parameters $\alpha, \beta, \gamma$ represent the degree of impact of the movement. If one of them does not exist in partial differential equations, the corresponding influence parameters are not affected by the presence of any of them. Most filters are required to have two images, one is the initial level set 
function model and one is the feature image to be segmented. We must specify the initial value of the level set function to represent the surface of the initial level set function. When the level set function is stopped, the output of the filter is the output of the filter. We need to get the image of the segmentation of the contour is obtained by the level set function of the surface of the zero level set. In general, the level set function is usually represented by the symbol distance function.

\section{Medical image registration algorithm based on TIK}

Image registration is a process of a space transformation, and the points in one image are mapped to the corresponding points in the other image. In ITK, registration is a framework that can be substituted for each functional component in the framework. This flexibility allows users to choose the most appropriate model to handle specific applications.

Here we choose a multimode registration method, which is carried out by experiments. Through the process and results of the test on the registration of ITK function, make an analysis. Multimode registration is a difficult problem in medical image registration, which means that the image is obtained by different acquisition devices. Therefore, simple application based on the direct comparison of the gray level of metrics is not feasible. It is now common view that the estimation of mutual information is more suitable for solving the problem of multimode image registration.

We adopt a registration algorithm called viola-wells mutual information. The algorithm uses the ITK mutual information image to image metric filter as feature space Metric. The gray value of the input image is adjusted by the filter. The purpose is to make the gray distribution of the input image into a zero mean and unit variance. It use ITK gradient descent optimizer filter as the optimizer, used to optimize Metric.

The algorithm of feature space Metric module of the to set several parameters, including for fixed image density estimation of Gaussian template standard difference for motion image density estimation of standard template, as well as for density and moisture of the sample size calculation. According to experience, we choose to estimate the standard deviation of the mutual information template is 0.4 .

The input and output of the filter is connected with the pipeline structure. Then we need to define the sample values for metric calculations, which are usually defined as a fraction of the total pixels, i.e., the proportion of the total pixels. In fixed images, the minimum number of sample points can be set to $1 \%$ of the total number of pixels, the higher the value, can improve the smoothness of metric, but increased the calculation time. So we need to think about compromise. In practice, the value of the value set is usually high. Because the mutual information value is relatively large, it means that the registration is relatively good. So we need to maximize the value of mutual information.

\section{Conclusion}

Through a series of experiments and simple code, the paper further studies the ITK algorithm and ITK method. By studying the process of ITK, on the one hand, the method of medical image processing is studied. On the other hand, it improves the ability of using ITK, which lays the foundation for the future use of ITK for image processing. Through the research of ITK architecture, it also builds the foundation for the construction of multimode medical image processing platform. 


\section{Reference}

[1] Wang Luo,Hongliang Li,Guanghui Liu,King Ngi Ngan. Global salient information maximization for saliency detection[J]. Signal Processing: Image Communication . 2011 (3).

[2] Tianjie Li,Yuanyuan Wang. Biological image fusion using a NSCT based variable-weight method[J]. Information Fusion . 2010 (2).

[3] Sabalan Daneshvar,Hassan Ghassemian. MRI and PET image fusion by combining IHS and retina-inspired models[J]. Information Fusion . 2009 (2).

[4] Stefan Franz Nemec,Philipp Peloschek,Maria Theresa Schmook,Christian Robert Krestan,Wolfgang Hauff,Christian Matula,Christian Czerny. CT-MR image data fusion for computer-assisted navigated surgery of orbital tumors[J]. European Journal of Radiology . 2008 (2).

[5] Boris Escalante-Ramírez. The Hermite transform as an efficient model for local image analysis: An application to medical image fusion[J]. Computers and Electrical Engineering . 2007 (2).

[6] L. Yang,B.L. Guo,W. Ni. Multimodality medical image fusion based on multiscale geometric analysis of contourlet transform[J]. Neurocomputing . 2008 (1).

[7] YONG C,YOU H,CHAOLONG Y.CT and MRI imagefusion based on contourlet using a novel rule. Pro-ceedings of the 2nd International Conference on Bioin-formatics and Biomedical Engineering (ICBBE 2008) . 2008.

[8] N. Dalal,B. Triggs.Histograms of oriented gradients for human detection. Coference on Computer Vision and Pattern Recognition (CVPR) . 2005. 